\title{
EFFECT OF HEATING RATE DURING BAKING ON THE PROPERTIES OF CARBON ANODES USED IN ALUMINUM INDUSTRY
}

\author{
Yasmine Chamam $^{1}$, Duygu Kocaefe ${ }^{1}$, Yasar Kocaefe ${ }^{1}$, Dipankar Bhattacharyay ${ }^{1}$, \\ Brigitte Morrais $^{2}$ \\ ${ }^{1}$ UQAC/AAI Research Chair on Carbon and REGAL Aluminum Research Center \\ University of Quebec at Chicoutimi 555 Boulevard de l'Université, Chicoutimi, QC, Canada G7H 2B1
}

${ }^{2}$ Aluminerie Alouette Inc., 400, Chemin de la Pointe-Noire, Sept-Îles, Québec, Canada G4R 5M9

Keywords: Carbon anodes, anode baking, heating rate, soaking time

\begin{abstract}
Carbon anodes are called the heart of aluminum electrolysis cells and contribute to about $15 \%$ of total production cost of aluminum. In case of poor quality, this contribution can reach $25 \%$. The final quality of the carbon anodes and their behavior in the electrolysis cells depend on the conditions during the baking process. In any case, a low-quality green anode cannot be improved during baking. However, the quality of green anodes can deteriorate if the parameters of the baking process are not properly adjusted.
\end{abstract}

The objective of this work is to study the effect of introducing a plateau during baking on the properties of carbon anodes. The samples were characterized before and after baking by measuring their density and electrical resistivity which are important in defining the final quality of the anode samples. The experimental data were also analysed using the artificial neural network model (ANN). The article will present the results of this study.

\section{Introduction}

Carbon anodes are used in Hall-Heroult cells to produce aluminum. Calcined petroleum coke, butts and rejected anodes are crushed to obtain a desired particle size distribution. These are then mixed with coal tar pitch to obtain a carbonaceous paste. The paste is then compacted and baked to produce baked anodes with appropriate properties such as high density, low electrical resistivity, and low air and $\mathrm{CO}_{2}$ reactivities. Increasing anode quality helps decrease energy consumption, production cost as well as greenhouse emissions.

Baking is by far the costliest stage in the manufacturing of anodes [1]. The final quality of the carbon anodes and their behavior in the electrolysis cells depend on the conditions of the baking process.

A few researchers investigated the effect of baking parameters on the anode quality. Belitskus [2] studied the effect of the final anode baking temperature on the quality of anodes and found that it has a major effect on anode electrical resistivity and excess anode consumption during electrolysis. The same parameter was also studied by Fischer et al. [3]. They found that low final baking temperatures or short soaking times lead to selective air and $\mathrm{CO}_{2}$ burning of the anodes in the electrolytic cells. This problem can be detected by an increase in the standard deviations of the reactivities. They recommended the utilization of baking temperatures in the range of $1050^{\circ} \mathrm{C}$ to $1200^{\circ} \mathrm{C}$ to optimize the $\mathrm{CO}_{2}$ and air reactivities and the thermal conductivity of anodes. They also pointed out the importance of heating rate on anodes. Their results showed that the presence of high anode temperature gradients significantly increases the standard deviations of electrical resistivity and flexural strength; hence, this results in nonhomogeneous anode properties. They recommended that the maximum heating rate should not exceed 10 to $14^{\circ} \mathrm{C} / \mathrm{h}$ between $200-600^{\circ} \mathrm{C}$ to control the devolatilization of tar components from pitch.

Severo et al. [4] studied the influence of the final baking temperature on the energy consumption. They found that increasing the baking temperature by $50^{\circ} \mathrm{C}$ increases the energy consumption by about $0.2 \mathrm{GJ} / \mathrm{t}$ of anodes.

Amrani et al. [5] showed that crack formation increases at high heating rates. Kocaefe et al. [6] reported that the baked anode properties such as air permeability as well as air and $\mathrm{CO}_{2}$ reactivities are improved if the anodes are baked at low heating rates.

Although a low heating rate leads to an increase in baking time, several researchers have strongly recommended their utilization to obtain high quality anodes. Keller et al. [7] mentioned that minimizing energy consumption should not be the goal of the baking process. It should be the improvement of the anode quality. They also suggested that a good control of the burning of pitch volatiles can minimize the energy consumption and the emission of greenhouse gases during the baking process.

In this study, the effect of the presence of a plateau at different temperatures during baking on the quality of anodes is investigated. Figure 1 shows qualitatively an example of a plateau during anode baking.

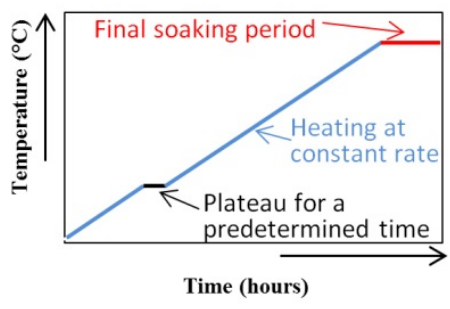

Figure 1 : Figure showing the plateau and the soaking time 
Due to large variations in the standard variations in the data analysed, the experimental data were also analysed using an artificial neural network (ANN) model.

ANN is a tool, often used in the field of artificial intelligence, to find the relation between a set of independent parameters and an output parameter where no straight forward mathematical relationship exists. Researchers have described feedforward artificial neural network with sigmoidal transfer function and backpropagation training as a universal function approximator [8]. A multi-layered feed forward ANN model was developed.

\section{Methodology}

Four sets of cylindrical samples (130 mm in length and 50 $\mathrm{mm}$ in diameter) were taken from the top and the bottom parts of two different industrial anodes (Anode 1 and Anode 2). They were baked at a heating rate similar to those typically used in industry in a laboratory baking furnace at the University of Quebec at Chicoutimi (UQAC).

A plateau for a predetermined time period was tested at different temperatures $\left(200^{\circ} \mathrm{C}<\mathrm{T} 1<\mathrm{T} 3<\mathrm{T} 5<\mathrm{T} 7<600^{\circ} \mathrm{C}\right)$ using different samples. This temperature range coincides with the devolatilization range of nearly all pitch volatiles [3]. Also, all anode samples were maintained at the maximum baking temperature for eight hours (final soaking time). Four samples from each anode were baked for each condition studied. The densities and electrical resistivities of the samples were measured before and after baking. Table 1 presents the specifications of the two industrial anodes used. The percentages of pitch in both anodes were nearly the same.

Table 1 : Specification of the industrial anodes

\begin{tabular}{|c|c|c|}
\hline & Anode 1 & Anode 2 \\
\hline Type of coke & $\begin{array}{c}\text { Coke 1 55\% } \\
\text { Coke 2 45\% }\end{array}$ & $\begin{array}{c}\text { Coke 3 low S 30\% } \\
\text { Coke 4 high S 70\% }\end{array}$ \\
\hline Type of pitch & Pitch 1 & $\begin{array}{c}\text { Pitch 1 90\% } \\
\text { Pitch 2 10\% }\end{array}$ \\
\hline
\end{tabular}

Figure 2 shows the experimental system. The anode samples were placed in packing coke, and nitrogen was passed through the system to prevent their oxidation. The furnace was programmed to have the desired heating rate. A thermocouple was placed in the packing coke, and the temperature was monitored during the experiment using a data acquisition system. After baking, the anode samples were cooled down to the room temperature under nitrogen atmosphere.

\section{$\underline{\text { Measurement and representation of anode properties }}$}

The bulk density and the electric resistivity of the anodes were measured before and after baking using the ASTM standards $[9,10]$. The bulk density was measured according to ASTM D5502-00 (2005). The apparent density of the cylindrical core samples of carbon anodes can be determined with this standard. The electrical resistivity was measured according the ASTM D6120-97 (2007). This test method is used for the determination of the electrical resistivity of cylindrical anode samples at room temperature.

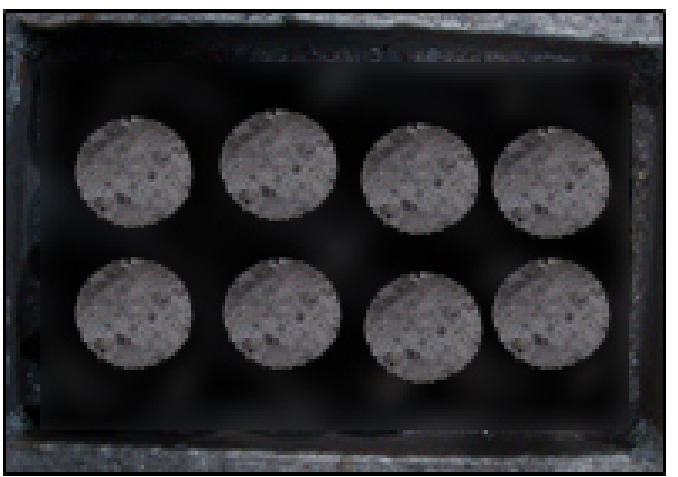

Figure 2: Placement of samples in the laboratory baking furnace

In this work, the densities of green and baked anode samples are expressed in dimensionless form using equation 1:

$$
D D=(G A D \text { or } B A D) / A V D
$$

where $\mathrm{DD}$ is the dimensionless density, GAD is the green anode density, BAD is the baked anode density, and AVD is the the average of the experimental values of GAD and BAD of all samples.

The green anode resistivities are expressed in dimensionless form using equation 2 :

$$
D G R=G A R / A V G R
$$

where DGR is the dimensionless green anode resistivity, GAR is the green anode resistivity, and AVGR is the average of the experimental values of GAR of all samples.

Similarly, the baked anode resistivities are expressed in dimensionless form using equation 3 :

$$
D B R=B A R / A V B R
$$

where DBR is the dimensionless baked anode resistivity, BAR is the baked anode resistivity, and AVBR is the average of the experimental values of BAR of all samples.

\section{Results and Discussion}

Eight samples (four from each anode) were baked under the same baking condition to account for the non-homogeneity of the industrial anodes in each case.

Figures 3 and 4 show the average dimensionless densities and resistivities of the green anode samples, respectively. These samples were later baked to study the impact of adding a plateau during the devolatilization period while the anodes are baked. The dimensionless values were 
calculated as explained above (Equations (1) and (2)). "No plateau" implies that no plateau was present when these samples were later baked. "Plateau Ti" implies that the baking temperature was maintained constant at this temperature for a predetermined time during the baking of these samples.

The standard deviations of density and resistivity of the green anode samples show that there was a significant variation in the properties of the green anodes used for the analysis. The variations in the density can be explained in terms of the non-homogeneity of the anodes with respect to the nature and distribution of particles, binder pitch and pores/cracks. To compare the effect of having a plateau at different temperatures, it is ideally necessary to use identical green anode samples with the same density and electrical resistivity. However, this is not possible when industrial anodes are used. Thus, as explained previously, a number of samples were used to test each condition.

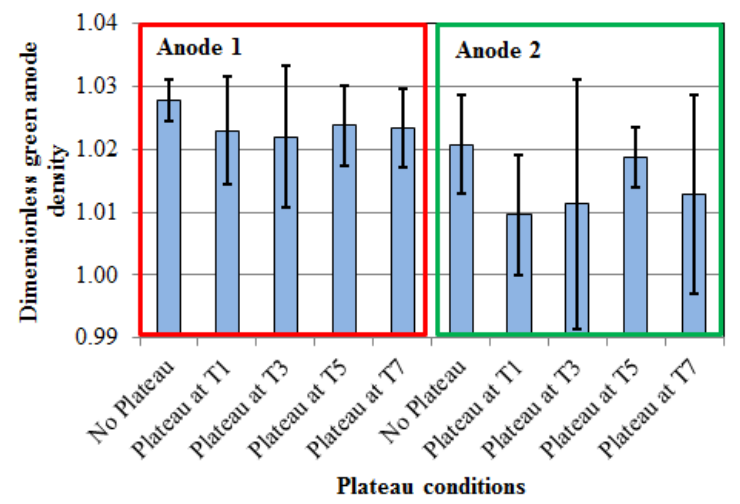

Figure 3: The average dimensionless densities and the standard deviations of the green anode samples

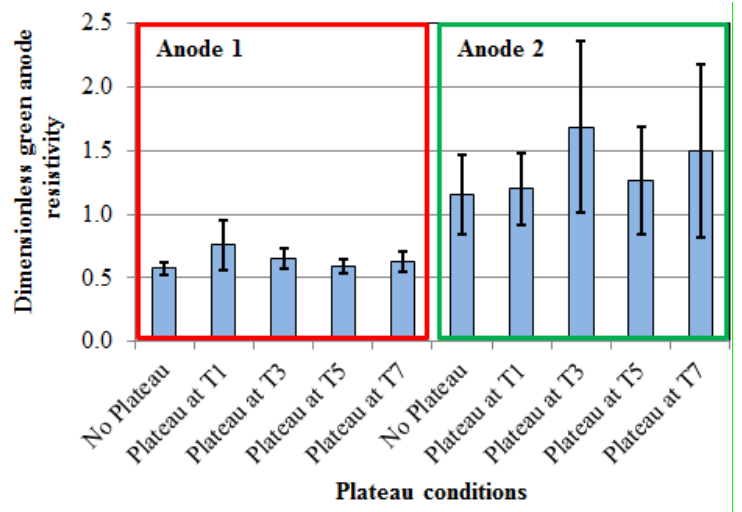

Figure 4: The average dimensionless electrical resistivities and the standard deviations of the green anode samples

As the initial values of the densities and resistivities of the green anode samples were different, the ratio of BAD to GAD for the density and the ratio of BAR to GAR were used to understand the effect of plateau temperature on the anode properties. The ratio shows how much a property is improved. Since a high BAD is required, a high $\mathrm{BAD} / \mathrm{GAD}$ is desirable. On the other hand, a low BAR is desirable. Thus, a low BAR/GAR value indicates good quality anode.

Figures 5 and 6 present the ratios of BAD/GAD and BAR/GAR for samples from anodes 1 and 2. The objective is to identify the plateau which will give high $\mathrm{BAD} / \mathrm{GAD}$ and low BAR/GAR ratios. It can be seen from these figures that this objective is reached for both anodes, around T7. This suggests that, by introducing a plateau during the devolatilization period, it might be possible to produce baked anodes with higher density and lower electrical resistivity than those baked without a plateau.

The standard deviations in Figures 5 and 6 show that the variations in $\mathrm{BAD} / \mathrm{GAD}$ and $\mathrm{BAR} / \mathrm{GAR}$ depend on the variations in green anode properties. As described earlier, it is difficult to find identical green anode samples. Thus, the artificial neural network (ANN) [11] was used to predict the density and resistivity of baked anode samples with identical GAD and GAR.

In this work, the experimental data for both anodes were used to train the ANN model. The model was validated with a set of experimental data which was not used for training. The output of the model was in good agreement with the experimental results.

This model was used to predict the BAD and BAR corresponding to the top and bottom parts of Anode 1 and Anode 2. ANN is based on pattern identification and recognition. The predicted properties should be in the range for which experimental data are available (T1 to T7). Thus, the BAD and BAR were predicted for a particular value of GAD and GAR corresponding to plateau temperatures of T1, T2, T3, T4, T5, T6, and T7. The chosen plateau temperatures are the averages of the experimental ones on either side of the temperature used for prediction in ANN as given below:

$\mathrm{T} 2=(\mathrm{T} 1+\mathrm{T} 3) / 2, \mathrm{~T} 4=(\mathrm{T} 3+\mathrm{T} 5) / 2, \mathrm{~T} 6=(\mathrm{T} 5+\mathrm{T} 7) / 2$

The output parameters BAD and BAR for different anode positions (top and bottom) are predicted based on the pattern identified from the experimental data set during the training phase of the ANN model.

Figures 7 and 8 show the predicted BAD and BAR corresponding to bottom and top parts of Anode 1, respectively, at different plateau temperatures. The predicted $\mathrm{BAD}$ and $\mathrm{BAR}$ at different plateau temperatures at the bottom and top of Anode 2, respectively, are presented in Figures 9 and 10.

It can be observed from Figures 7-10 that the predicted BAD and BAR varied at the top and bottom positions of the two anodes for the same GAD and GAR. The predictions of the model varied based on the pattern identified in the training data set. The figures also showed that at around temperature $\mathrm{T} 6$, the predicted $\mathrm{BAD}$ is the maximum and BAR is the minimum. The predictions of ANN can be improved if more experimental data is available. 


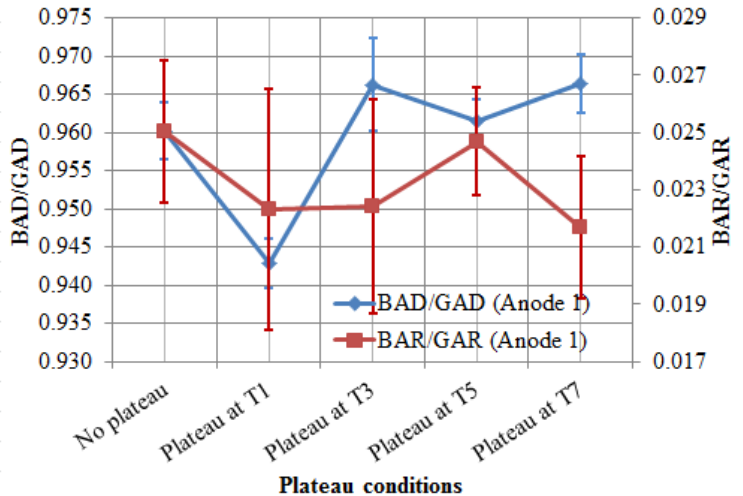

Figure 5: Ratios of BAD/GAD and BAR/GAR for Anode 1 for different baking conditions (without plateau and with plateau at different temperatures)

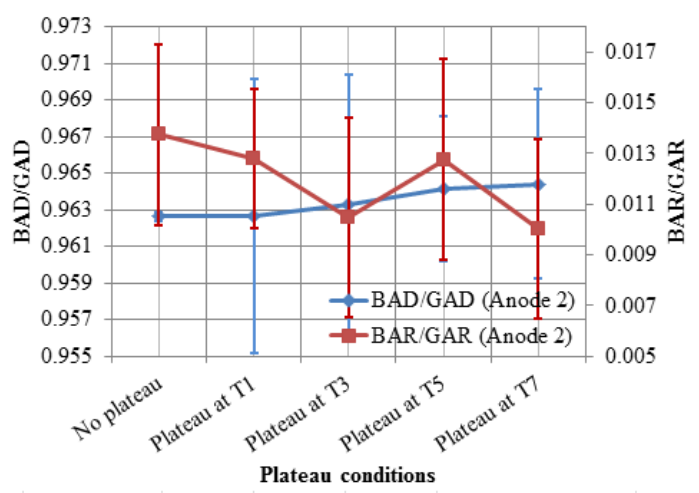

Figure 6: Ratios of BAD/GAD and BAR/GAR for Anode 2 for different baking conditions (without plateau and with plateau at different temperatures)

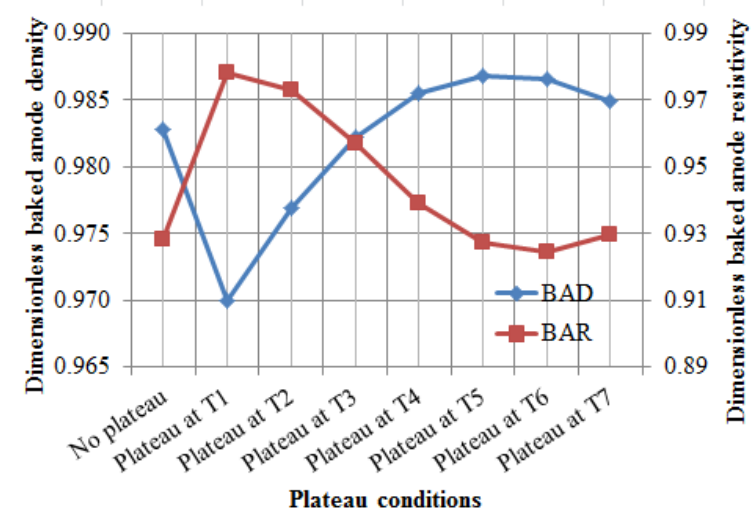

Figure 7: Predicted BAD and BAR for samples from the bottom part of Anode 1 for constant GAD and GAR

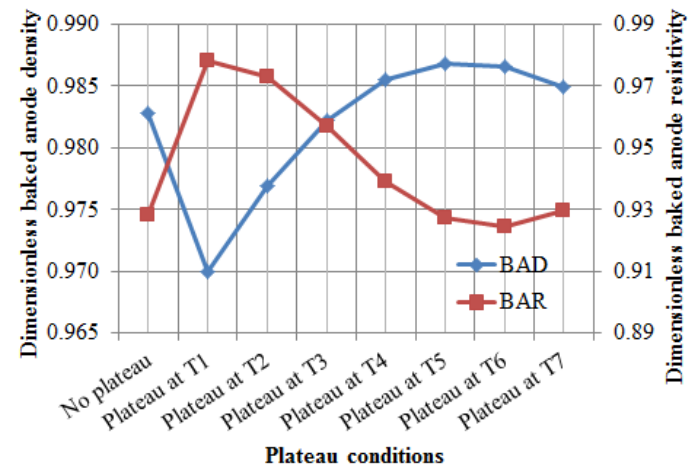

Figure 8: Predicted BAD and BAR for samples from the top part of Anode 1 for constant GAD and GAR

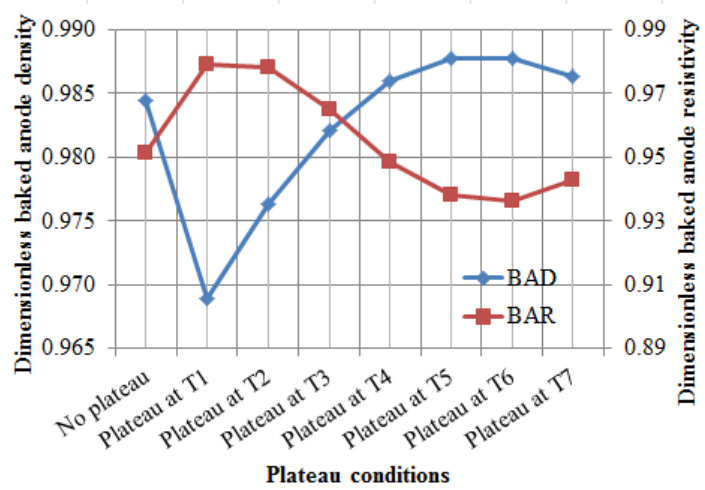

Figure 9: Predicted BAD and BAR for samples from the bottom part of Anode 2 for constant GAD and GAR

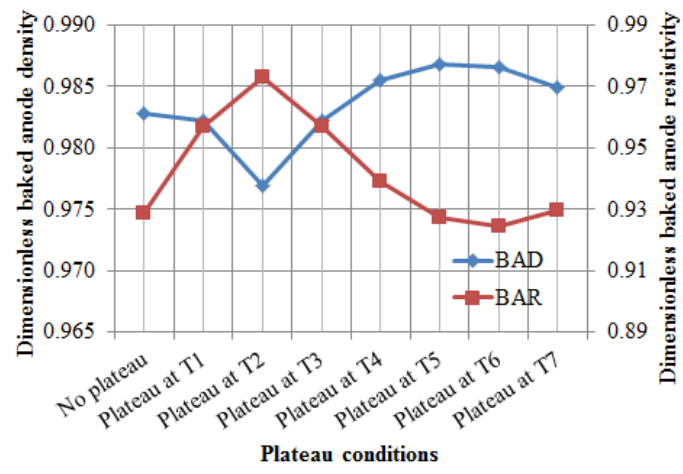

Figure 10: Predicted BAD and BAR for samples from the top part of Anode 2 for constant GAD and GAR 
It may be noted that no experiment was performed at the plateau temperature of T6 exactly. The ANN model and the experimental data analyses showed that plateau temperatures of $\mathrm{T} 6$ and T7, respectively, might improve the baked anode density and resistivity. These two temperatures are very close. The ANN and the experimental data analyses identified similar plateau temperatures. It is possible that adding a plateau in this temperature range would seem to help control the rate of volatiles release from pitch during baking consequently improve the baked anode quality.

\section{Conclusions}

Anode quality is crucial for aluminum smelters and anode baking is an important step in producing good quality anodes. The baking process influences the anode quality as well as the production and operation costs. In this work, the effect of adding a plateau at a certain temperature during the devolatilization period was studied. The ratios obtained from the experimental data for BAD/GAD and BAR/GAR as well as the results from the artificial neural network analysis showed that introducing such a plateau could improve the baked anode quality.

Similar plateau temperatures were obtained from both analyses. The study indicated that a good quality baked anode with high density and low electrical resistivity can be produced if the plateau is set in the range of T6 and T7 for a predetermined time.

\section{Acknowledgements}

The technical and financial contributions of Aluminerie Alouette Inc. and the financial support from the Natural Sciences and Engineering Research Council of Canada (NSERC), the Economic Development Sept-îles, the University of Quebec at Chicoutimi (UQAC), and the Foundation of the University of Quebec at Chicoutimi (FUQAC) are greatly appreciated.

\section{References}

[1] Keller, F.,Oderbolz, S. Process Controlled Operation of Baking Furnaces, light Metals 1985, 1107-1123.

[2] Belitskus, D.L. An evaluation of relative effects of coke, formulation, and baking factors on aluminium reduction cell anode performance. Light Metals 1993, 677681.

[3] Fischer, W.,K., Keller, F, Perruchoud, R., Oderbolz, S. (1993). Baking parameters and the resulting anode quality. Light Metals 1993, 683- 689.

[4] Severo, D., Peter Sulger,P., Keller, F., Meier, M. Recent Developments in Anode Baking Furnace Design, Light Metals 2011, 853 - 858

[5] Amrani, S., Kocaefe, D., Kocaefe, Y., Morais, B., Blaney, G.. Effect of heating rate on the crack formation during baking in carbon anodes used in aluminium industry, Light Metals 2014, 1175-1180.

[6] Kocaefe, D., Xie, Y., Kocaefe, Y., Wei, L., Zou, S., $\mathrm{Wu}$, A. Thermogravimetric Study on Devolatilization Kinetics of Cinalco anodes during Baking. Journal of materials Science Research 2013, Vol 2, No. 2, 22-34.

[7] Felix Keller, Peter Sulger, Markus Meier, Dagoberto Severo, Vanderlei Gusberti, "Specific Energy Consumption in Anode Bake Furnaces”, Light Metals 2010, 1005 - 1010

[8] White, H. Artificial neural networks: Approximation and Learning Theory, Blackwell, Cambridge, 1992.

[9] ASTM D5502-00 (2010). Standard Test Method for Apparent Density by Physical measurements of manufactured Anode and Cathode Carbon Used by the Aluminium Industry, ASTM International, 1225-1226.

[10] ASTM D6120-97 (2007). Standard Test Method for Electrical Resistivity of Anode and Cathode Carbon Material at Room Temperature, ASTM International 320322.

[11] Bhattacharyay, D., Kocaefe, D., Kocaefe, Y., Morais, B., Comparison of Linear Multivariable, Partial Least Square Regression and Artificial Neural Network Analyses to Study the Effect of Different Parameters on Anode Properties, Light Metals, 2015. 